\title{
Nonlinear Normalization Model to Improve the Performance of Neural Networks
}

\author{
Daehyon Kim¹)
}

\begin{abstract}
An intelligent transportation system (ITS) generally contains an automatic traffic video-surveillance system as a primary subsystem. Such subsystem incorporates the capabilities of neural networks for the efficient and effective recognition and classification of complicated spatial and temporal patterns in real-world traffic scenarios. Notably, the properties of input vectors are the key factors in determining the performance of neural networks. These properties are governed by the method used to normalize these vectors; a simple linear scaling model is widely employed for normalizing input vectors. This study proposes the use of a nonlinear normalization model for input vector normalization. The proposed technique is subsequently applied to neural networks to resolve classification problems encountered when analyzing real-world traffic image data. The experimental results show that the proposed model can produce higher prediction accuracy, when compared to the existing linear-based approach models. This model has the potential to improve the performance in traffic machine vision applications.
\end{abstract}

Keywords: Intelligent Transportation Systems (ITS), automatic traffic surveillance, neural networks, input vector normalization, traffic machine vision

\section{Introduction}

Automatic video-based traffic surveillance analysis is one of the major research topics in the domain of intelligent transportation systems (ITS), and a significant amount of research has been dedicated to improve their prediction accuracy[1-3]. However, the accuracy is still not satisfactory in the case of complicated circumstances, and algorithms with increased efficiency must be developed to improve system reliability for traffic scene analysis[4]. Neural network models have been used throughout the past two decades for prediction and analysis in complex surroundings, largely because their remarkable ability to derive meaning from complicated or imprecise data.

Currently, multilayer feed-forward neural networks using the backpropagation learning algorithm is one of the most popular learning models. This learning algorithm has been widely

Received(June 20, 2020), Review Result(1st: August 10, 2020, 2nd: September 26, 2020), Accepted(October 28, 2020)

1) (Professor) 59626 Dept. of Culture and Tourism Management, Chonnam National University, Yeosu-Si, Jeollanam-Do, Korea

email: daehyon@chonnam.ac.kr 
applied to various problems[5-7], including traffic scene analysis. Recently, deep neural networks (DNNs), a feed-forward neural network with multiple hidden layers, have emerged as a powerful machine learning model[8-10].

In order to use backpropagation neural networks and DNNs appropriately, several major issues such as the network topology, input and output vector normalization, and optimal initial weights should be considered first before applying the model to real-world data. The normalization requirements for the input and output vectors of the neural network has been theoretically studied [11]. The study also showed that an input vector normalization method could affect the predictive performance of the neural network model in a pattern recognition problem. Currently, the most common methods for normalizing input vectors are linear-based models. Although the simple linear models are widely used, the nonlinear models can be more efficient and effective for input and output vector normalization.

In this paper, a nonlinear normalization method is proposed in order to achieve improved prediction accuracy. The predictive performance of an existing linear-based approach and the proposed nonlinear-based model has been evaluated by comparing their ability to classify real-world traffic image data.

\section{Normalization for Input Vector and Neural Networks}

The input vectors of neural network models and learning algorithms such as the backpropagation learning algorithm[12], DNNs[13], and support vector machines (SVMs)[14] must be normalized before being used in the models. Otherwise, the network training process can undergo failure because several hidden neurons (or units) have fixed values. Carpenter and Grossberg[15] proposed a simple normalization method for the application of a Fuzzy ARTMAP neural network, which is a combination of fuzzy logic and the adaptive resonance theory (ART) neural network. Normalization in the Fuzzy ARTMAP can be achieved in a straightforward manner using the following formula.

$$
\tilde{x}=\frac{\bar{x}}{|x|}
$$

,where $\bar{x}=\left(x_{1}, x_{2}, \ldots, x_{n}\right)$ is the incoming vector with $\mathrm{n}$ representing the number of input units/vectors, and the norm $|\cdot|$ can be expressed as $|x|=\sum_{i}\left|x_{i}\right|$. However, in Eq. (1), if there are several input vectors, i.e., i.e. $n$ (the number of input units) is very large, then the denominator is also very large; this results in saturation. To address this, Kim[11] suggested a 
new linear normalization method that may produce improved vehicle detection performance through traffic surveillance image processing.

To date, several neural network models and learning algorithms have been proposed. However, the multilayer feed-forward network using backpropagation is the most popular neural network model because it is easy to implement and widely applicable. It has been shown to produce relatively good results in many applications. In this study, an advanced backpropagation neural network, in which a weight-decay parameter was added to the algorithm involving backpropagation with momentum, was used to evaluate the normalization methods. The multilayer feed-forward neural network using the backpropagation learning algorithm can be summarized as [16]:

Step 1: Configure the input, output vectors, and network architecture.

Step 2: Randomize the initial weights.

Step 3: Calculate the output values for each unit of the network by using:

$$
O u t_{p k}^{l}=f_{p k}^{l}\left(N e t_{p k}^{l}\right), \text { where } N e t_{p k}^{l}=\sum_{j=1}^{K_{l-1}} w_{k j}^{l} I_{p j}^{l}+\theta_{k}^{l}
$$

In Eq. (2), $I_{p j}^{l}$ are the inputs to the $k^{t h}$ unit in layer $l, \mathrm{w}$ is the number of synaptic weights in the network, $\theta_{k}^{l}$ is a bias term, $K_{l}$ is the number of $l$ llayer neurons, $p$ is a training pattern, and $f()$ is an activation function.

Step 4: For the output layer, $l=L$, calculate the values of the weight changes by using:

$$
\Delta_{p} w_{k j}^{L}=\eta \delta_{p k}^{L} I_{p j}^{L}, \text { where } \delta_{p k}^{L}=\left(y_{p k}-O u t_{p k}^{L}\right)\left(f_{k}^{L}\left(N e t_{p k}^{L}\right)\right)^{\prime}
$$

In Eq. (3), $y_{p k}$ is the desired output.

Step 5: For the hidden layers, $l=1,2, \ldots, L-1$, calculate the weight change values using:

$$
\Delta_{p} w_{j u}^{l}=\eta \delta_{p j}^{l} I_{i j}^{l}, \text { where } \delta_{p j}^{l}=\left(f_{k}^{L}\left(N e t_{p k}^{L}\right)\right)^{\prime} \sum_{k=1}^{K_{l+1}} \delta_{p k}^{l+1} w_{k j}^{l+1}
$$

Step 6: Update the weights on the output layers by:

$$
w_{k j}^{L}(t+1)=w_{k j}^{L}(t)+\Delta_{p} w_{k j}^{L}
$$

Step 7: Update the synaptic weights on the hidden layers by:

$$
w_{j u}^{l}(t+1)=w_{j u}^{l}(t)+\Delta_{p} w_{j u}^{l}, l=1, \ldots, L-1
$$


Step 8: Repeat the previous steps (from Step 3 to Step 7) until the average squared error computed over the entire training data set is an acceptably small value. The error for the output units is then calculated by:

$$
E r r=\sum_{k=1}^{K_{L}}\left(y_{p k}-O u t_{p k}^{L}\right)^{2}
$$

\section{Linear and Nonlinear Models for Normalization}

\subsection{Linear Models for Normalizing Input Vectors}

The use of a maximum and minimum input vector value in each input pattern is one of the most prevalent normalization methods in which the input vectors are normalized. The formula for this normalization method is represented by Eq. (8). Using this method, each input vector value is within the range of 0.0 to 1.0 .

$$
\widetilde{a_{p i}}=\frac{a_{p i}-a_{p \min }}{a_{p \max }-a_{p \min }}
$$

,where $\quad a_{p \max }=\max \left(p_{p i} ; i=1,2, \ldots, m\right) \quad a_{p \min }=\min \left(a_{p i} ; i=1,2, \ldots, m\right), \quad$ and $p(p=1,2, \ldots, P)$ are the input patterns. In Eq. (8), $\widetilde{a_{p i}}$ denotes the normalized value of the unit $\mathrm{i}$ of the input vector. Additionally, $a_{p \mathrm{max}}$ and $a_{p \mathrm{~min}}$ are the maximum and minimum values, respectively, of the input unit across all the input patterns, i.e.: $a_{p \max }=\max \left\{p_{1}\left(a_{1}, a_{2}, \ldots, a_{m}\right), \ldots, p_{P}\left(a_{1}, a_{2}, \ldots, a_{m}\right)\right\}$ and

$a_{p \text { min }}=\min \left\{p_{1}\left(a_{1}, a_{2}, \ldots, a_{m}\right), \ldots, p_{P}\left(a_{1}, a_{2}, \ldots, a_{m}\right)\right\}$

, where $p_{1}\left(a_{1}, a_{2}, \ldots, a_{m}\right), \ldots, p_{P}\left(a_{1}, a_{2}, \ldots, a_{m}\right)$ are the input patterns. Currently, Eq. (8), which is a linear-based model is the most prevalent normalization method.

\subsection{Nonlinear Model for Normalizing Input Vectors}

Herein, we propose a sigmoid nonlinear model for normalizing input vectors. A sigmoid function is a mathematical function having an " $\mathrm{S}$ " shape (sigmoid curve), as shown in Eq. (10).

$$
\widetilde{a_{p i}}=\frac{1}{1+\exp \left(-a_{p i}+C_{c}\right)}
$$


, where exp is the exponential function $a_{p i}$ is an independent variable (representing pixel grayscale in this study), and $C_{c}$ is a constant:

$$
C_{c}=\frac{\left(a_{p \max }-a_{p \min }\right)}{2}
$$

, where $a_{p \max }$ and $a_{p \min }$ are the maximum and minimum values, respectively, of the input units across all input patterns. In Eq. (10), $C_{c}$ is a constant that is calculated using two fixed values from the data set. In this study, $C_{c}=128$, because $a_{p \max }=255$ and $a_{p \min }=0$.

The sigmoid function in Eq. (10) magnifies the values in the neighborhood of the median, $\mathrm{Cc}$, which is the numerical value separating the higher half of a data sample from the lower half. This normalization model tends to emphasize the median of the data sets. Conversely, this equation decreases the effect of high-level and low-level values near the maximum and minimum values of the data sets, respectively. The demagnification of the high-level and low-level values may lead to a reduction in noise in these levels owing to the smearing of noise in the data sets.

\section{Performance Evaluation of Normalization Models}

The input vector normalization method has a significant influence on the properties of the input vector. The shape of the distribution of the input vectors can be modified by the normalization methods. Both the learning and prediction accuracy of neural network models are influenced by changes in the distribution of the input vector projections. In this section, the performance of the proposed nonlinear model is compared with that of a standard linear model through numerous experiments, using real-world traffic surveillance analysis data.

\subsection{Application of Artificial Neural Networks}

In this study, a pattern recognition problem related to traffic scene analysis was considered to evaluate the network performance by comparing the results of different input vector normalization methods. The task involved the classification of three different patterns viz. Patterns A, B, and C (see [Fig. 1]). A large number of data sets used in this study for training and testing have also been used in previous research projects[17]. A total of 930 data sets were used for the experiments, including 400 sets for Pattern A, 400 sets for Pattern B, and 130 sets for Pattern $\mathrm{C}$. The data sets were randomly split into two subsets; one for training and the 
other for testing. The training subset contained 230 data sets and the test data subset contained 700 data sets.

[Fig. 1] also shows the two-dimensional distribution shape of the input vector projection according to the normalization method used. The $x, y$ coordinates of the figure are respectively, the horizontal and vertical addresses of each pixel and the z-axis is the intensity of the pixel. As shown in the figure, the linear function of Eq. (8) creates a linear mapping between two modules (or vector spaces) and maintains the same morphology. However, a nonlinear normalization model creates nonlinear mappings that nonlinearly transform the data in the original input space into the scaled data in the normalizing space, i.e., Eq. (10) increases the higher data values nonlinearly.

In order to evaluate the performance of the normalization method, the experiments were implemented using three-layer network architecture (450 - 450 - 3), with a learning rate of 0.2, momentum of 0.5 , weight decay of 0.1 , and a mini-batch size of 10 . The input vectors used in this research were 256 greyscale images, 15 by 30 pixels in size. For the output vectors, three units were used to recognize three different patterns, i.e. $y_{1}=\left[\begin{array}{lll}1 & 0 & 0\end{array}\right], y_{2}=\left[\begin{array}{lll}0 & 1 & 0\end{array}\right]$, and $y_{3}=\left[\begin{array}{lll}0 & 0 & 1\end{array}\right]$.

\subsection{Predictive Performance on Linear and Nonlinear Normalization}

It is known that the performance of the backpropagation model is sensitive to the initial weight configuration. In order to properly evaluate a model, many trials must be executed on the same network using the same parameter values but different initial weights. In this study, 30 trials were performed using different initial weights that were initialized to random values between -0.5 and +0.5 to avoid the effects of the initial weight values. The network was trained for 150 epochs to achieve 100\% recognition accuracy for the training set with a very low training error. More importantly, an analysis of variance (ANOVA) test was performed on the experimental results with a significance level of $\mathrm{P}=0.05$, to assess the statistical significance of the experimental results.

[Table 1] presents the results of the experiments performed using different input vector normalization methods in the multilayer feed-forward neural network using the backpropagation learning algorithm. The predictive performance of the linear and nonlinear normalization functions was compared using the best, worst, and average cases. [Table 1] lists the results of the two normalization methods, i.e., the linear and nonlinear models based on 30 trials. [Table 1](A) presents the best and worst predictive performance on the test sets and [Table 1](B) 
presents the average of the predictive performance on the test sets.

In [Table 1], in linear normalization that is the most common normalization method, the input vectors are normalized by Eq. (8) in a linear transformation. The prediction error rate in the table expresses the recognition error rate in the test sets (the number of errors/the number of test sets). The correct recognition rate on the test sets ranges from $82.4 \%$ to $85.6 \%$ according to the initial weights. In this linear normalization method, the average correct prediction rate from 30 trials (using the test sets) is $84.1 \%$, where the prediction error rate is $15.9 \%$.

However, the proposed nonlinear model, that includes a sigmoid function, yields predictive performance improvements in all cases, i.e., best, worst, and average. The non-linear normalization, in which the input vectors are normalized with the sigmoid function, produces improved performance in terms of the prediction accuracy, when compared to general linear normalization. When the input vectors are normalized by using a sigmoid function, the correct recognition rate on the test sets ranges from $83.9 \%$ to $88.6 \%$ according to the initial weights. Moreover, the average correct prediction rate from 30 trials (using the test sets) is $86.1 \%$, where the prediction error rate is $13.9 \%$.

The ANOVA, which is a statistical analysis tool, indicated significant differences in the predictive performance between the linear and non-linear normalization methods. As shown in [Table 1] (B), the P-value (2.64E-10), which is extremely small, indicates that the result is statistically significant and the difference in the mean of the two groups is real.

The overall results obtained in this study showed that the predictive performance of a model can be further improved by adapting a novel normalization method for the input vectors of the neural networks. Although linear models are widely used for input and output vector normalization, nonlinear models can be more powerful than their linear counterparts. Furthermore, the nonlinear model proposed in this study can also be applied to other problems and has the potential to improve the performance of other learning models, such as SVMs and DNNs.

Summary of the findings are as follows; (a) Experimental results show that we can achieve about $2.0 \%$ better prediction performance with nonlinear normalized model compared to a linear model in neural networks; i.e. the nonlinear normalized inputs reduces the prediction error rate from $15.9 \%$ to $13.9 \%$ and increases the recognition rate from $84.1 \%$ to $86.1 \%$. (b) ANOVA (statistical test) indicated that there was a significant difference between the two normalization methods, linear and nonlinear, and in all experimental results, the nonlinear model performed better than the linear model. 


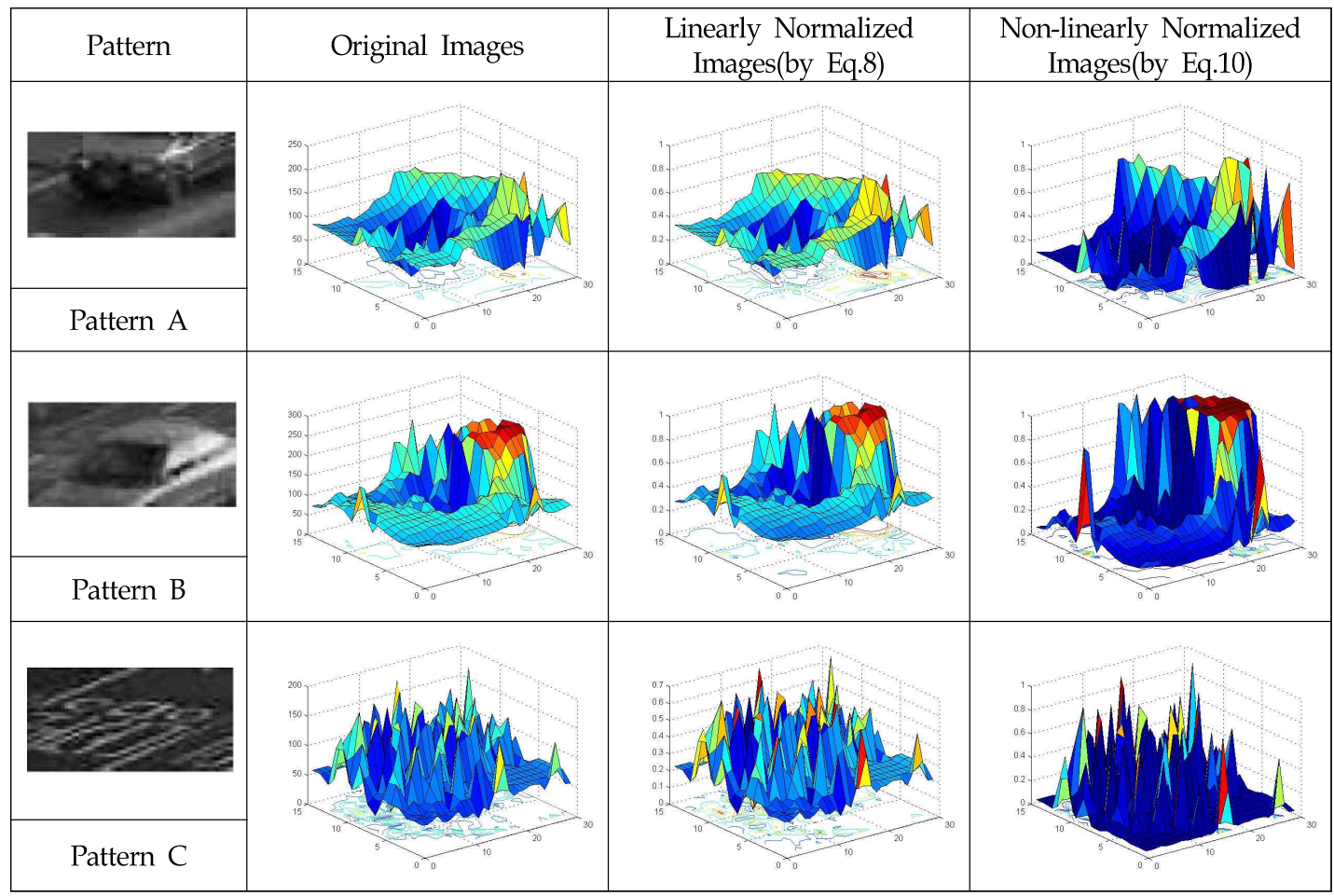

[Fig. 1] Two-dimensional distribution of normalized inputs of three patterns

[Table 1] Predictive Performance from Linear and Nonlinear Normalization (A) Best and Worst Predictive Performance on the Test Sets

\begin{tabular}{|c|c|c|c|c|c|}
\hline & \multicolumn{4}{|c|}{ Normalization Method } \\
\hline & & \multicolumn{2}{|c|}{ Linear Normalization } & \multicolumn{2}{|c|}{ Non-linear Normalization } \\
\hline & & Best Performance & Worst Performance & Best Performance & Worst Performance \\
\hline \multicolumn{2}{|c|}{ Training RMSE } & 0.00037 & 0.00038 & 0.00035 & 0.00039 \\
\hline \multirow{3}{*}{$\begin{array}{l}\text { Prediction } \\
\text { Errors }\end{array}$} & Pattern 1 & $14 / 300$ & $18 / 300$ & $25 / 300$ & $29 / 300$ \\
\hline & Pattern 2 & $87 / 300$ & $105 / 300$ & $49 / 300$ & $74 / 300$ \\
\hline & Pattern 3 & $0 / 100$ & $0 / 100$ & $6 / 100$ & $10 / 100$ \\
\hline \multicolumn{2}{|c|}{ Number of total errors } & $101 / 700$ & $123 / 700$ & $80 / 700$ & $113 / 700$ \\
\hline \multicolumn{2}{|c|}{ Prediction error rate $(\%)$} & 14.4 & 17.6 & 11.4 & 16.1 \\
\hline \multicolumn{2}{|c|}{ Recognition rate $(\%)$} & 85.6 & 82.4 & 88.6 & 83.9 \\
\hline
\end{tabular}

(B) Average of Predictive Performance on the Test Sets from 30 Trials

\begin{tabular}{c|c|c|c}
\hline \multirow{2}{*}{} & \multicolumn{2}{c}{ Normalization Method } \\
\cline { 2 - 4 } & Linear Normalization & Non-linear Normalization \\
\hline \multicolumn{2}{c|}{ Training RMSE } & 0.00038 & 0.00034 \\
\hline \multirow{3}{*}{ Prediction errors } & Pattern 1 & 15.03 & 24.93 \\
\cline { 2 - 4 } & Pattern 2 & 96.17 & 64.20 \\
\cline { 2 - 4 } & Pattern 3 & 0.0 & 8.27 \\
\hline \multicolumn{2}{c|}{ Number of total errors } & 111.20 & 97.4 \\
\hline \multicolumn{2}{c}{ Prediction error rate (\%) } & 15.9 & 13.9 \\
\hline \multicolumn{2}{c}{ Recognition rate (\%) } & 84.1 & 86.1 \\
\hline \multirow{2}{*}{ Statistical analysis } & Std. Deviation & 5.37 & 8.33 \\
\cline { 2 - 4 } & Groupings & B & $\mathrm{A}$ \\
\cline { 2 - 4 } & P-value & & $2.64 \mathrm{E}-10$ \\
\hline
\end{tabular}


Note: Each value on this table is an average of 30 trials.

\section{Conclusions}

The input and output vectors in neural network models must be normalized if the values of the input vectors are not within the range of 0 to 1 (or -1 to 1 ). The normalization of the input vectors causes the original data properties to change and the predictive performance to be affected, but most studies involving the use of neural networks have not investigated the normalization methods.

This study proposed a new normalization method for input vectors based on nonlinear mapping. The predictive performance of the general linear-based model, which is currently the most common model, was compared to that of the nonlinear-based model using real-world traffic scene analysis, in which each model detects the vehicle image patterns.

The experimental results showed that the proposed nonlinear-based model for input vector normalization can produce higher predictive accuracy than the most widely used linear normalization method. Thus, the results of this study recommend the use of a nonlinear normalization model to increase the performance of the neural network model. The proposed normalization method may also be applied to other problems and may be able to provide higher predictive performance for other neural network models.

\section{References}

[1] W. Dong, Z. Yang, W. Ling, Z. Yonghui, L. Ting, Q. Xiaoliang, Research on vehicle detection algorithm based on convolutional neural network and combining color and depth images, IEEE 2nd International Conference on Information Systems and Computer Aided Education (ICISCAE), (2019), September 28-30; Dalian, China, DOI: 10.1109/ICISCAE48440.2019.221634

[2] L. W. Tsai, J. W. Hsieh, K. C. Fan, Vehicle detection using normalized color and edge map, IEEE Transaction on Image Processing, (2007), Vol.16, No.3, pp.850-864, DOI: 10.1109/TIP.2007.891147

[3] R. O'Malley, M. Glavin, E. Jones, Vision-based detection and tracking of vehicles to the rear with perspective correction in low-light conditions, IET Intelligent Transport Systems, (2011), Vol.5, No.1, pp.1-10, DOI: 10.1049/iet-its.2010.0032

[4] D. Kim, Prediction Performance of Support Vector Machines with Fused Data in Road Scene Analysis, International Journal of Transportation, (2015), Vol.3, No.3, pp.41-48, DOI: 10.14257/ijt.2015.3.3.04

[5] A. Mukherjee, D. K. Jain, P. Goswami, Q. Xin, L. Yang, J. Rodrigues, Back Propagation Neural Network Based Cluster Head Identification in MIMO Sensor Networks for Intelligent Transportation Systems, IEEE Access, (2020), Vol.8, 28524-28532, DOI: 10.1109/ACCESS.2020.2971969 
[6] S. Wang, X. Liu, Y. Jin, K. Qu, Wind Power Short Term Forecasting based on Back Propagation Neural Network, International Journal of Smart Home, (2015), Vol.9. No.7, pp.231-240.

[7] Y. Li, Exploration Analysis Based on Improved BP Neural Network in Human Body Movement, International Journal of Multimedia and Ubiquitous Engineering, (2016), Vol.11, No.7, pp.331-338, DOI: 10.14257/ijmue.2016.11.7.33

[8] D. Kim, J. Seok, Active Sonar Target Classification Using Multi-aspect based Sensing and Deep Belief Network, Asia-Pacific Journal of Neural Networks and Its Applications, (2018), Vol.2, No.2, pp.1-6, DOI: 10.21742/ajnnia.2018.2.2.01

[9] P. Chandra, A Survey on Deep Learning its Architecture and Various Applications, Asia-Pacific Journal of Neural Networks and Its Applications, (2017), Vol.1. No.2, pp.7-14, DOI: 10.21742/ajnnia.2017.1.2.02

[10] J. Schmidhuber, Deep learning in neural networks: An overview, Neural Networks, (2015), Vol.61, pp.85-117, DOI: 10.1016/j.neunet.2014.09.003

[11] D. Kim, Normalization Methods for Input and Output Vectors in Backpropagation Neural Networks, International Journal of Computer Mathematics, (1999), Vol.71, No.2, pp.161-171, DOI: $10.1080 / 00207169908804800$

[12] D. E. Rumelhart, J. L. McClelland, A General Framework for Parallel Distributed Processing, Parallel Distributed Processing, MIT Press, (1986), pp.45-76.

[13] G. E. Hinton, S. Osindero, Y-W. Teh, A Fast Learning Algorithm for Deep Belief Nets, Neural Computation, (2006), Vol.18, No.7, pp.1527-1554, DOI: 10.1162/neco.2006.18.7.1527

[14] C. Cortes, V. Vapnik, Support vector networks, Machine Learning, (1995), Vol.20, pp.273-297, DOI: https://doi.org/10.1007/BF00994018

[15] G. A. Carpenter, S. Grossberg, Fuzzy ARTMAP: Supervised learning, recognition, and prediction by a self-organizing neural network, IEEE Communications Magazine, (1992), Vol.30, No.9, pp.38-49, DOI: $10.1109 / 35.156802$

[16] D. Kim, Acquiring Real Time Traffic Information Using Deep Learning Neural Networks, Asia-pacific Journal of Multimedia Services Convergent with Art, Humanities, and Sociology, (2016), Vol.6, No.5, pp.435-444, DOI: 10.35873/ajmahs.2016.6.5.042

[17] D. Kim, Pre-processing of inputs to a neural network model for better performance in traffic scene analysis, Civil Engineering and Environmental Systems, (2010), Vol.27, No.1, pp.23-31, DOI: 10.1080/10286600802252719 